\title{
Post-Extrasystolic Changes of the Vectorcardiographic T Loop in Healthy Subjects
}

\author{
VN Batchvarov ${ }^{1}$, II Christov ${ }^{2}$, G Bortolan $^{3}$, II Simova ${ }^{4}$, AJ Camm ${ }^{1}$ \\ ${ }^{1}$ St. George's University of London, London, United Kingdom \\ ${ }^{2}$ Centre of Biomedical Engineering, Bulgarian Academy of Sciences, Sofia, Bulgaria \\ ${ }^{3}$ Institute of Biomedical Engineering ISIB - CNR, Padova, Italy \\ ${ }^{4}$ University Hospital 'Aleksandrovska', Clinic of Cardiology, Sofia, Bulgaria
}

\begin{abstract}
Visible T wave changes of the $1^{\text {st }}$ sinus beat following a ventricular premature beat (VPB) are sometimes observed in the electrocardiograms (ECG) of both healthy subjects and cardiac patients. We hypothesized that ventricular repolarization of the post-VPB beat can be modulated even without visible $T$ wave changes. We analyzed 12-lead ECGs acquired in 7 healthy subjects, with at least $1 \mathrm{VPB} / E C G$ with no visible changes of the $1^{\text {st }}$ post-VPB beat. The spatial T loop of each sinus beat was reconstructed in derived $X Y Z$ leads and was characterized by 5 parameters. The $1^{\text {st }}$ post-VPB beat was compared with the $2^{\text {nd }}$ and $3^{\text {rd }}$ pre-VPB beats. In 6 subjects, 1 to 3 T-loop parameters were significantly modified with an average change from $-18.9 \%$ to $30.1 \%$ signifying increased repolarization heterogeneity. In conclusion, heterogeneity of the post-VPB sinus beat can be increased in the absence of visible $T$ wave changes.
\end{abstract}

\section{Introduction}

Visible changes in the $\mathrm{T}$ (or T-U) wave of the sinus beat immediately following a ventricular premature beat (VPB) are not infrequently observed in electrocardiograms (ECG) of both healthy subjects and cardiac patients (for a recent review see [1]). Their mechanism is not yet fully understood. It is possible, however, that ventricular repolarization of the $1^{\text {st }}$ sinus complex is modulated even in the absence of visible to the naked eye $\mathrm{T}$ wave changes.

Quantitative analysis of the $\mathrm{T}$ wave morphology has been shown to provide information about the heterogeneity of ventricular repolarization, which can not be obtained by visual inspection of the $\mathrm{T}$ wave of the standard 12-lead ECG, or measurement of simple parameters such as QT dispersion. Various methods for T wave morphology analysis have been proposed, such as analysis of area-derived parameters [2], algebraic decomposition of the $\mathrm{T}$ wave [3], principal component analysis [4,5], analysis of the T loop using singular value decomposition [6] and others.

Another possibility is to analyze quantitatively the vectorcardiographic (VCG) T loop. Although the VCG contains the same information as the scalar ECG, its specific form of presentation of the ECG signal allows the detection of abnormalities that are not immediately visible on the scalar ECG. In this study, we implemented a previously reported method of quantitative analysis of the vectorcardiographic T-loop [7-9] in order to compare sinus complexes preceding and immediately following VPBs. We analyzed standard 12-lead ECGs acquired in subjects with no heart disease and frequent VPBs.

\section{Methods}

\subsection{ECG database}

Digital 10-second 12-lead ECGs (MAC5000, GE Medical Systems, Milwaukee, WI, USA, $500 \mathrm{~Hz}, 12$ bit A/D) acquired in 7 healthy subjects ( 3 men, 4 women, age 35.3 \pm 7.9 years, range $23-48$ years) were selected from the ECG database of the Department of Cardiac and Vascular Sciences at St. George's, University of London. All subjects had negative medical history and normal physical examination, except for the presence of frequent premature beats and were not taking any medications. In each subject, 10 consecutive ECGs were acquired in the resting supine position. All ECGs were normal except for the presence of frequent monomorphic VPBs with no visible changes in the $T$ wave of the $1^{\text {st }}$ post-VPB sinus 
beat. ECGs with at least 3 sinus beats preceding and 2 sinus beats following a VPB were selected for further analysis (5.3 \pm 0.8 , range 5-7 ECGs per subject). They were converted into text files (Magellan ECG Research Workstation programme, GE medical Systems) which were then transferred into a custom-made software programme [9] for analysis of the VCG T loop.

\subsection{Quantitative analysis of VCG T-loop}

The spatial T-loop of the vectorcardiogram (VCG) was synthesized using orthogonal Frank leads derived from the standard 12-lead ECG. Several methods for derivation of Frank orthogonal leads from simultaneously recorded 12 standard leads have been proposed [10]. In this study, we used the method of Levkov [11,12].

The QRS and T loop have generally the form of an ellipse, which is best characterized by its major axis. For example, Nowinski et al. [13] defined the $\mathrm{T}$ loop eigenvalue parameter as a ratio of the major to the minor axis of the ellipse. In previously published studies [7-9], the VCG T-loop was characterized by parameters which were measured (i) towards the maximal axis of the ellipse, and (ii) towards the isoelectric (zero) point of the ECG. The former approach was shown to provide superior results [7-9] and therefore in this study the Tloop parameters were measured with respect to the maximal axis of the ellipse.

The following 5 parameters were analysed:

- Angle in the T-loop in the frontal plane (Tf)

- Angle in the T-loop in the horizontal plane (Th)

- Maximum angle between QRS and T loop axes (MA)

The angles $\left(A_{F}, A_{H}\right.$ and $\left.A_{L S}\right)$ in the three VCG planes - frontal, horizontal and left sagittal - are computed, and the maximum value is taken as parameter MA:

$$
\mathrm{MA}=\max \left(\mathrm{A}_{\mathrm{F}}, \mathrm{A}_{\mathrm{H}}, \mathrm{A}_{\mathrm{LS}}\right)
$$

- $T$ axis elevation and azimuth angle difference (DEA). This parameter is adopted from the work of Kallert et al. [14]. The difference between frontal plane elevation and azimuth angles is calculated along the entire segment of repolarization, providing a description of the T-wave loop morphology. DEA is defined as the mean absolute value of the difference between the frontal plane Elevation $(\alpha)$ and Azimuth $(\beta)$ of all loop samples (n)

$$
D E A=\operatorname{mean}\left(a b s\left(\alpha_{n}-\beta_{n}\right)\right)
$$

- The Elevation with respect to the frontal plane is the angle between the loop axis at sample $n$ and the $\mathrm{Y}$ axis in the left sagittal plane (Fig. 1(a)), while the Azimuth is the angle between the loop axis at the same sample and the $\mathrm{X}$ axis in the frontal plane (Fig. 1(b)).

- Ratio of maximum to mean $T$ vector magnitudes
$(R M M V)$. This ratio is adopted from the work of Kallert et al. [14]:

(a)

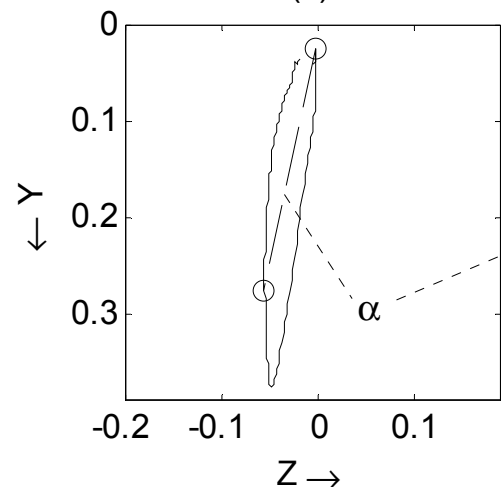

(b)

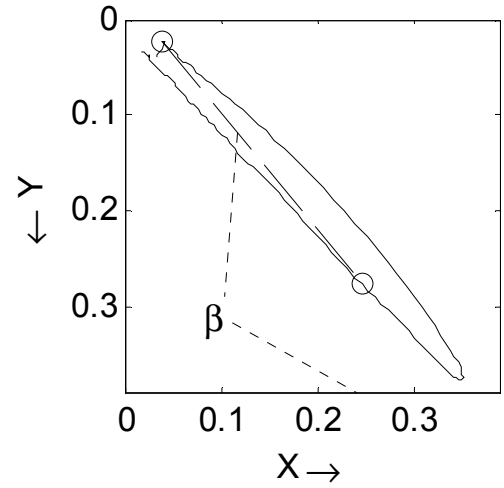

Figure 1. Measurement of $\mathrm{T}$ axis Elevation $(\alpha)$ and Azimuth $(\beta)$ at the $(n)$ sample of the loop

$\mathrm{RMMV}=\mathrm{V}_{\max } /$ mean $\left(\mathrm{V}_{\mathrm{n}}\right)$,

where $V_{n}$ is the magnitude of the spatial vector of the $\mathrm{T}$ loop at sample $\mathrm{n}$, calculated by the formula:

$\mathrm{V}=\sqrt{\mathrm{V}_{\mathrm{X}}^{2}+\mathrm{V}_{\mathrm{Y}}^{2}+\mathrm{V}_{\mathrm{Z}}^{2}}$,

where $\mathrm{V}_{\mathrm{X}}, \mathrm{V}_{\mathrm{Y}}$ and $\mathrm{V}_{\mathrm{Z}}$ are the magnitudes of the projection of the spatial vector on the orthogonal axes.

Increase in MA and DEA, Tf and Th and decrease in RMMV signify abnormal inscription of the T-loop and hence increased heterogeneity of ventricular repolarization. Abnormal inscription of the T-loop has previously been reported in patients with ischaemic heart disease compared to healthy controls [9].

\subsection{Statistical analysis}

For each subject and each parameter, the $1^{\text {st }}$ post-VPB complex was compared with the average of the $2^{\text {nd }}$ and $3^{\text {rd }}$ pre-VPB complexes (unpaired t-test). Data are presented as mean \pm standard deviation (SD). A P value of $<0.05$ was considered to signify statistically significant difference. 


\section{Results}

In 6 out of 7 subjects, 1 to 3 of the T loop parameters were significantly modified (Table 1).

Table 1 Significant changes of the T loop parameters in the individual subjects

\begin{tabular}{cccc}
\hline \hline Parameter & Pre-VPB & Post-VPB & P value \\
\hline & $302.40 \pm 1.19$ & $297.80 \pm 2.59$ & 0.007 \\
$\operatorname{Tf}\left(\mathrm{n}=3^{*}\right)$ & $316.25 \pm 2.30$ & $309.43 \pm 3.05$ & 0.0009 \\
& $265.17 \pm 49.86$ & $303.50 \pm 3.87$ & 0.03 \\
\hline Th $\left(\mathrm{n}=1^{*}\right)$ & $10.3 \pm 2.2$ & $13.4 \pm 2.6$ & 0.02 \\
\hline & $31.83 \pm 3.08$ & $28.00 \pm 1.26$ & 0.02 \\
MA $\left(\mathrm{n}=3^{*}\right)$ & $19.92 \pm 1.07$ & $14.83 \pm 2.48$ & 0.001 \\
& $140.94 \pm 19.15$ & $115.60 \pm 6.72$ & 0.0011 \\
\hline DEA $\left(\mathrm{n}=1^{*}\right)$ & $59.75 \pm 5.29$ & $69.33 \pm 8.02$ & 0.035 \\
\hline RMMV & $2.91 \pm 0.19$ & $2.70 \pm 0.06$ & 0.031 \\
$\left(\mathrm{n}=2^{*}\right)$ & $2.52 \pm 0.09$ & $2.10 \pm 0.16$ & $4 \times 10^{-6}$ \\
\hline \hline
\end{tabular}

$*=$ number of subjects with statistically significant changes; pre$\mathrm{VPB}=$ average value for the $2^{\text {nd }}$ and $3^{\text {rd }}$ pre-VPB sinus complex; post-VPB = value for the $1^{\text {st }}$ post-VPB sinus complex

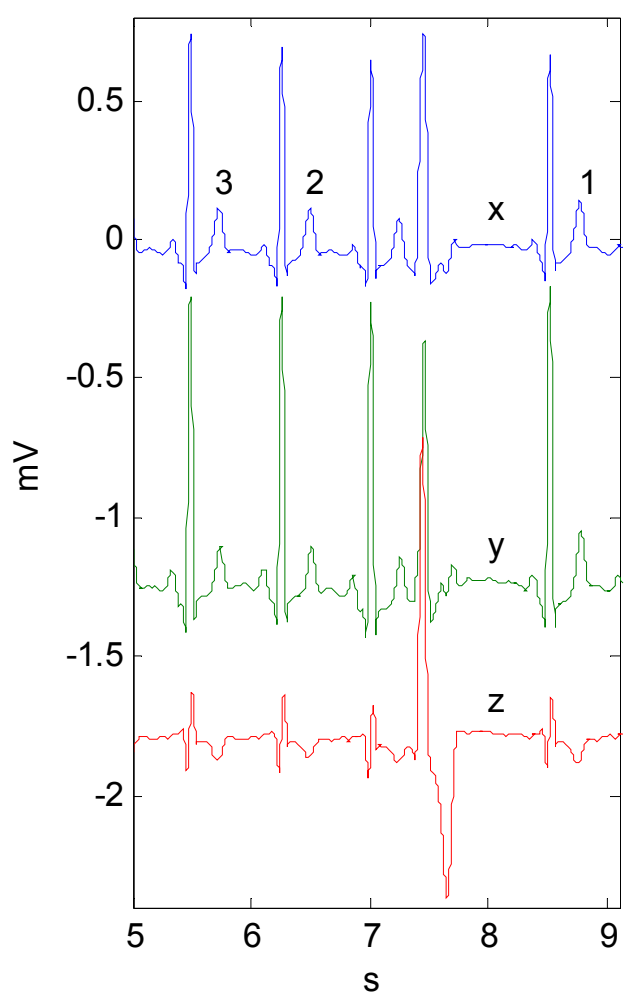

Figure 2. Frank orthogonal leads (x, y, z) obtained from the 12 Standard leads. Two pre-extrasystolic T-waves are numbered with "2" and " 3 ". The post-extrasystolic Twave is numbered with " 1 ".
An example of post-extrasystolic change of the T-loop and the ECG in orthogonal XYZ leads are presented in Figure 2, Figure 3 and Table 2

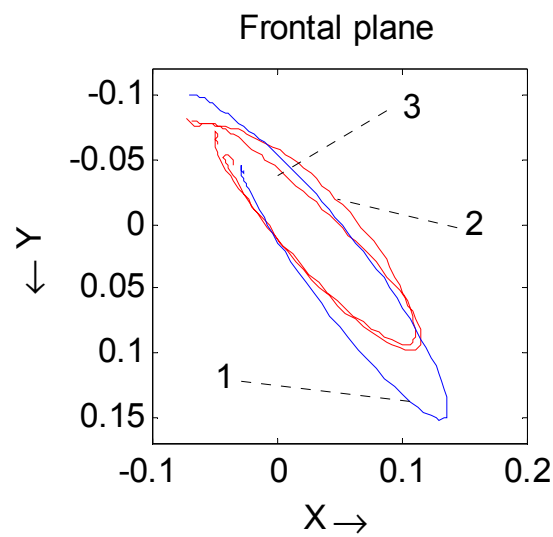

Figure 3. Vector T-loops in the frontal plane. The two pre-extrasystolic T-waves are numbered with " 2 " and " 3 ", and the post-extrasystolic T-wave is numbered with " 1 ".

Table 2. Vectorcardiographic parameters obtained for the 3 T-loops, shown in Fig. 3.

\begin{tabular}{cccccc}
\hline \hline No & Tf & Th & MA & DEA & RMMV \\
\hline 3 & 316 & 18 & 28 & 48 & 2.6 \\
2 & 316 & 17 & 27 & 48 & 2.7 \\
1 & 309 & 18 & 28 & 50 & 2.4 \\
\hline
\end{tabular}

\section{Discussion}

Our results demonstrate that in healthy subjects, ventricular repolarization of the $1^{\text {st }}$ sinus beat immediately following a VPB can be modulated, and the repolarization heterogeneity is considerable even in absence of visible changes of the $\mathrm{T}$ wave of the 12-lead ECG. At least theoretically, this modulatory influence may have potentially arrhythmogenic effect.

Changes in the $\mathrm{T}$ wave amplitude, shape or direction of the sinus beat immediately following a ventricular premature beat (VPB) were first reported by P.D. White in 1915 [15] and repeatedly by other authors thereafter [1619]. They can be observed in both healthy subjects, as well as cardiac patients and their association with the presence or severity of cardiac disease is unclear. Their mechanism is still not fully understood [1]. It has been suggested that in cases when the vector of the post-VPB $\mathrm{T}$ wave mirrors the general direction of the ectopic QRS complex (the so-called "obedient" $\mathrm{T}$ wave changes), the phenomenon likely represents a form of short-term 
cardiac memory [20]. Such T wave changes are not pause dependent (e.g. they can follow an interpolated VPB [1]). In contrast, the "non-obedient" $T$ wave changes (i.e. when the post-extrasystolic $\mathrm{T}$ wave vector is not concordant with the ectopic QRS), which are pause-related or depend on the coupling interval of the VPB are probably due to other mechanisms [1].

At present, our knowledge about the clinical significance of post-VPB repolarization changes seems to be limited only to the role of post-VPB and post-pause T and $\mathrm{U}$ wave augmentation in the "climate of torsades de pointes" [1] for the initiation of the arrhythmia [21].

In this study, we did not assess the relation between the post-VPB repolarization change and the direction of activation of the ectopic complex, the duration of the coupling interval/ post-ectopic pause, or the site of origin of the VPB.

In conclusion, our results suggest that the postextrasystolic repolarization modulation is very likely more frequent than previously appreciated and therefore deserves further studies in both healthy subjects and patients with various cardiac diseases.

\section{References}

[1] Childers R. Influence of Rhythm Abnormalities on Ventricular Repolarization. In: Malik M, Camm AJ (editors): Dynamic Electrocardiography, $1^{\text {st }}$ edition, Futura Blackwell Publishing, Oxford 2004:390-405.

[2] Merri M, Benhorin J, Alberti M, Locati E, Moss AJ. Electrocardiographic Quantitation of Ventricular Repolarization. Circulation 1989; 80:1301-1308.

[3] Padrini R, Butrous G, Camm AJ, Malik M. Algebraic Decomposition of the TU Wave Morphology Patterns. Pacing Clin Electrophysiol 1995; 18:2209-2215.

[4] Priori S, Mortara D, Napolitano C, et al. Evaluation of the Spatial Aspect of the T-Wave Complexity in the Long-QT Syndrome. Circulation 1997; 96:3006-3012.

[5] Badilini F, Fayn J, Maison-Blanche P, et al. Quantitative Aspects of Ventricular Repolarization: Relationship Between Three-Dimensional T Wave loop Morphology and Scalar QT Dispersion. A.N.E. 1997; 2:146-157.

[6] Acar B, Yi G, Hnatkova K, et al. Spatial, temporal and wavefront direction characteristics of 12-lead $\mathrm{T}$ wave morphology. Med Biol Eng Comput 1999; 37:574-584.

[7] Bortolan G, Bressan M, Christov I. Gender and age influences in T-loop morphology, Computers in Cardiology 2003;30:513-516.

[8] Bortolan G, Bressan M, Christov I. Longitudinal modifications of the T-loop morphology. Computers in Cardiology 2002;29:685-688.
[9] Bortolan G, Christov I. Myocardial infarction and ischemia characterization from T-loop Morphology in VCG. Computers in Cardiology 2001;28:633-636.

[10] Rubel P, Benhadid I Fayn J. Quantitative assessment of eight different methods for synthesising Frank VCGs from simultaneously recorded standard ECG leads. J. Electrocardiology, Suppl. 1992;24:197-202.

[11] Levkov C. Orthogonal electrocardiogram derived from the limb and chest electrodes of the conventional 12-lead system. Med. \& Biol. Eng. \& Comp. 1987;25:155-164.

[12] Daskalov IK, Dotsinsky IA and Christov II. Developments in ECG acquisition, preprocessing, parameter measurement and recording. IEEE Eng. in Med. \& Biol. 1998;17:50-58.

[13] Nowinski K, Jensen S, Lundahl $G$ and Bergfeldt L. Changes in ventricular repolarization during percutaneous transluminal coronary angioplasty in humans assessed by QT interval, QT dispersion and T vector loop morphology. Journ. of Intern. Med. 2000;248:126-136.

[14] Kallert T, Couderc JP, Voss A, Zareba W. Semi-automatic method quantifying $\mathrm{T}$ wave loop morphology: Relevance for assessment of heterogeneous repolarization. Comp. in Card. 1999;26:153-156.

[15] White PD. Alternation of the Pulse: A Common Clinical Condition. Am J M Sc. 1915; 150:82.

[16] Katz LN, Langendorf R, Cole SL. The unusual effect of interpolated ventricular premature systoles. Am Heart J 1944; 28:167-176.

[17] Scherf D. Alterations in the form of the $T$ waves with changes in heart rate. Am Heart J 1944; 28:332-347.

[18] Levine HD, Lown B, Streeper RB. The Clinical Significance of Postextrasystolic T-Wave Changes. Circulation 1952; 6;538-548.

[19] Mann RH, Burchell HB. The significance of T-wave inversion in sinus beats following ventricular extrasystoles. Am Heart J 1954: 47:504-513.

[20] Rosenbaum MB, Blanco HH, Elizari MV. Electrocardiographic characteristics and main causes of pseudoprimary $\mathrm{T}$ wave changes. Significance of concordant and discordant $\mathrm{T}$ waves in the human and other animal species Ann NY Acad Sci 1990; 601:36-50.

[21] Jackman WM, Friday KJ, Anderson JL, Aliot EM, Clark M, Lazzara R. The Long QT Syndromes: A Critical Review, New Clinical Observations and a Unifying Hypothesis. Prog Cardiovasc Dis 1988; XXXI:115-172.

Address for correspondence:

Dr. Velislav Batchvarov, MD

Division of Cardiac and Vascular Sciences,

St. George's, University of London,

Cranmer Terrace, London SW17 0RE,

United Kingdom.

e-mail: vbatchva@sgul.ac.uk 